\title{
TOPOSES, MONOID ACTIONS, AND UNIVERSAL COALGEBRA
}

\author{
ROBERT C. DAVIS
}

\begin{abstract}
A category cotripleable over Sets may be a topos, or may fail to be a topos in at least two distinct ways. One class of examples involves the category of $M$-sets and "strong" homomorphisms. For finite monoids $M$, this category is cotripleable iff the left ideals of $M$ are totally ordered by inclusion.
\end{abstract}

Introduction. Categories cotripleable over Sets satisfy some of the conditions needed to be elementary toposes - they have left and right limits and disjoint coproducts. Yet it is not obvious how the two concepts relate to each other. One does have Theorem 1 on p. 215 of [1], but it is not generally applicable here since the functor part of the cotriple is rarely left exact. Looking more closely, one would expect that the subobject classifier $\Omega$ should be derived from $G(2)$, where $G$ is the right adjoint, by excising from one of the two compartments all but a copy of the terminal object $T=G(1)$ and taking the largest subobject of what remains. However, to prove the pullback clause in the definition of a subobject classifier, one must assume:

(1) all monos are one-to-one;

and to prove the uniqueness clause, one must assume:

(2) the set-theoretic inverse image of any subobject is a subobject.

Finally, to have a topos, there is the requirement:

(3) exponential objects exist.

All three of these conditions can fail in a cotripleable category. It seems to be an open question whether (2), (3) and cotripleability together imply (1), but we will show by example that (2) and (3) may fail independently. However, many of the naturally occurring cotripleable categories are toposes, so by way of contrast we begin with a few examples to indicate some of the variety of phenomena that can occur. (All examples known to us are Grothendieck toposes; whether this is always the case seems to be an open question, though it is easy to find examples of cotripleable categories which are not toposes and which do not have a set of generators.) One of the counterexamples will be a case of the category $S(M)$ of $M$-sets and strong homomorphisms introduced in [4]. It was pointed out there that $S(M)$ is always quasicotripleable, and the question was raised as to when it is actually cotripleable, i.e. when the underlying-set functor has a right adjoint $G$. It turns out that, for finite $M$, this condition is equivalent to a structural property of $M$ that would at first sight seem to be totally unrelated.

Received by the editors January 15, 1985 and, in revised form, March 25, 1985 and May 20, 1985.

1980 Mathematics Subject Classification. Primary 18C15; Secondary 18B25.

Key words and phrases. Cotripleable category, right adjoint, terminal object, elementary topos, subobject classifier, exponential object, monoid, left ideal, $\mathcal{L}$-class. 


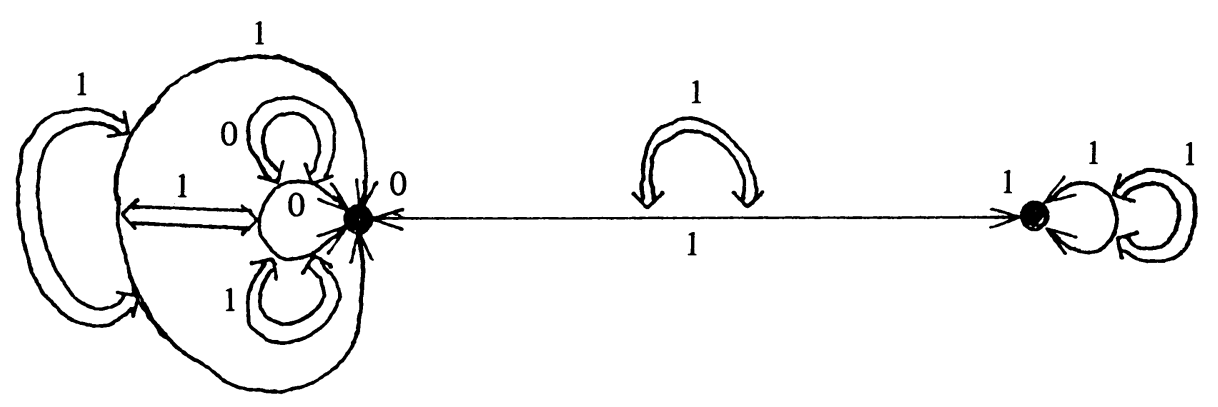

FIGURE 1

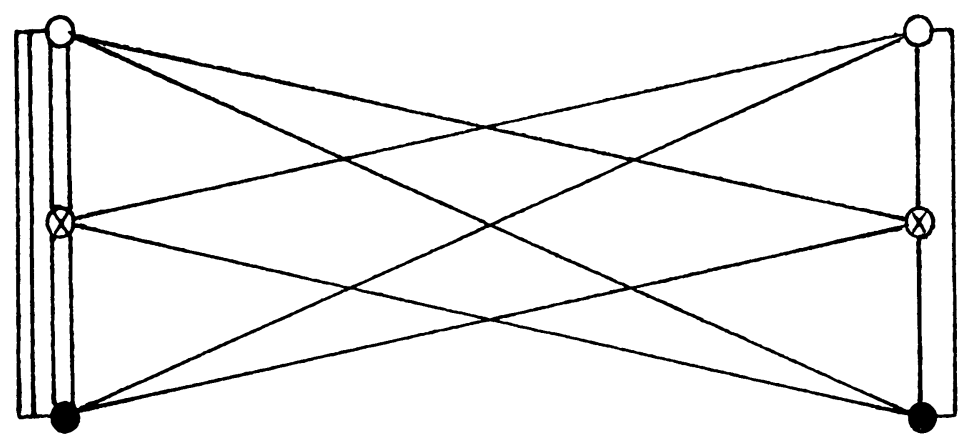

FIGURE 2

EXAMPLES. Simple examples of cotripleable toposes include the category of $M$ sets and ordinary homomorphisms for any monoid $M$, the example at the top of $\mathrm{p}$. 333 of [4], and the category of multigraphs (with loops) described in Theorem 1 of [3]. Others include:

1. The category of $Z_{2}$-sets which are also multigraphs, under the compatibility conditions that edges can only connect points interchanged by the $Z_{2}$ action.

2. The category of directed multigraphs, with homomorphisms that preserve both incidence and the orientation of the edges. As in all such examples, the underlying set includes both the vertices and the edges.

3. The generalization of multigraphs to higher dimensions. In two dimensions, any two coterminous edges (possibly identical) may be joined by one or more 2cells, and homomorphisms preserve dimension and incidence. Here, $G(2)$ has two vertices, six edges, and eighteen 2-cells; $T$ has one element of each dimension and can be seen embedded in the left-hand side of $\Omega$, which looks like Figure 1. All this works similarly for the $r$-dimensional analogue, in which $G(n)$ has $n+n\left(n+\left(\begin{array}{l}n \\ 2\end{array}\right)\right)+$ $n\left(n+\left(\begin{array}{c}n \\ 2\end{array}\right)\right)^{2}+\cdots+n\left(n+\left(\begin{array}{c}n \\ 2\end{array}\right)\right)^{r}$ elements.

4. The category of $r$-colored multigraphs, where the vertices are colored in $r$ colors and any two vertices joined by an edge must be colored differently. For $r=2$ one has the category of bipartite multigraphs. The homomorphisms are to preserve both incidence and color. For example, if $r=3, G(2)$ has six vertices, two of each color, and 24 edges; $T$ is a triangle, and can be seen in the left-hand side of $\Omega$, which looks like Figure 2. In general $G(n)$ has $r n+\left(\begin{array}{l}r \\ 2\end{array}\right) n^{3}$ elements. 
Counterexamples. 1. An example which violates condition (2) of the introduction alone is given by the category of multigraphs without loops described on $\mathrm{p}$. 33 of [3]. For example, there is a homomorphism

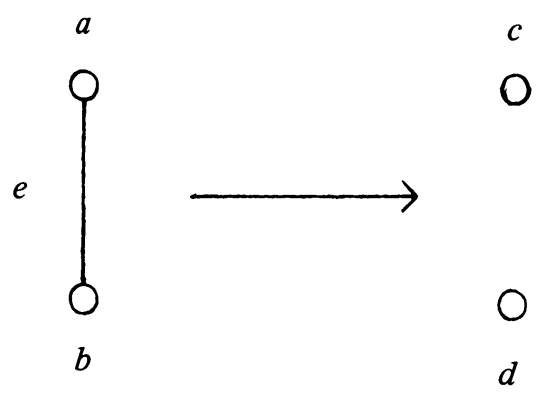

taking $a$ and $b$ to $c, e$ to $d$, and then the inverse image of the subgraph $d$ is $e$, which is not a subgraph.

2. An example violating condition (3) alone is given by the category described on the bottom of p. 333 of [4]. If $M$ is a monoid and $A, B$ are $M$-sets, a homomorphism $f: A \rightarrow B$ is called strong if it satisfies the additional condition that $f(a)=m b$ implies $a=m c$ for some $c$ such that $f(c)=b$. $M$-sets and strong homomorphisms form a category $S(M)$; for $M=\{e, m\}$ with $m^{2}=m$, it was pointed out in [4] that $S(M)$ is cotripleable. In this case, let $M$-sets $X, Y$ be defined by $X=Y=\{1,2, b\}$, where $m(1)=m(2)=b$. We will show that $Y^{X}$ does not exist. Let $Z$ consist of a set $A$ sent by $m$ to a base point not in $A$. Then each component of the categorical product $Z \prod X$ consists of a set $B \subseteq A \times\{1,2\}$ sent by $m$ to a base point. There is one such component for each $B$, subject to the condition that the projections send $B$ onto both $A$ and $\{1,2\}$. (For example, if $A$ has three elements, then $Z \prod X$ has 25 components and 127 elements.) Thus, the number of components is at least $2^{|A|}$, and each one admits more than two (strong) homomorphisms onto $Y$, so the number of homomorphisms $Z \prod X \rightarrow Y$ is at least $2^{2^{|A|}}$. Now, if $Y^{X}$ existed and had cardinal $\kappa$, the number of homomorphisms $Z \rightarrow Y^{X}$ would be at most $\kappa^{|A|}$. But, for large enough $A$, one has

$$
2^{2^{|A|}}>\kappa^{|A|}
$$

so a one-to-one correspondence is impossible and $Y^{X}$ does not exist.

When is $S(M)$ cotripleable? The first answer we can give to this question is, "not always." Let $N$ be the additive monoid of the nonnegative integers, so an $N$-set is just a set $A$ with a map $f: A \rightarrow A$. Then we have:

LEMMA. $S(N)$ is not cotripleable.

PROOF. We show that the terminal object $T$ does not exist. Supposing it does, let $B$ be the set of all the connected components of $T$ which end in a single fixed point. One can now joint all of the components in $B$ to a new common fixed point, redefining $f$ so as to take the previous fixed points to the new one. As the components were nonisomorphic and strongly irreducible, so must the new structure be irreducible. One can then repeat the entire process, but with an arbitrary subset of $B$ in place of $B$. This yields a collection of $|P(B)|$ nonisomorphic irreducible components, a contradiction. 
Note that this result implies that $S(N)$ has arbitrarily large irreducible objects (having no proper strong homomorphic images), a fact we shall use shortly.

We will call a monoid $M$ left ordered if its principal left ideals are totally ordered by inclusion. That is, $M$ is left ordered iff, for any $x, y$ in $M$, there is $z$ in $M$ such that either $x=z y$ or $y=z x$. In terms of the multiplication table of $M$, this means that either $x$ occurs in the $y$ column or $y$ occurs in the $x$ column, so the condition is easily checked for small $M$. Then we have the following result; the preceding lemma shows that it cannot be extended without alteration to infinite monoids.

THEOREM. For a finite monoid $M$, the following conditions are equivalent:

1. $S(M)$ is cotripleable;

2. $S(M)$ has a terminal object;

3. $M$ is left ordered.

Proof. $1 \Rightarrow 2$ : Trivial, since $T=G(1)$.

$2 \Rightarrow 3$ : Suppose $M$ is not left ordered. Then there are $x, y$ in $M$ such that for no $z$ in $M$ do we have $x=z y$ or $y=z x$. Now we use each of the arbitrarily large irreducible $N$-sets provided by the lemma as a model for constructing an irreducible $M$-set. Each arrow $\circ \stackrel{f}{\rightarrow} \circ$ in the diagram of the $N$-set is replaced by

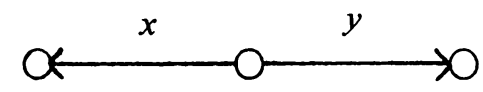

for example, to the $N$-set:

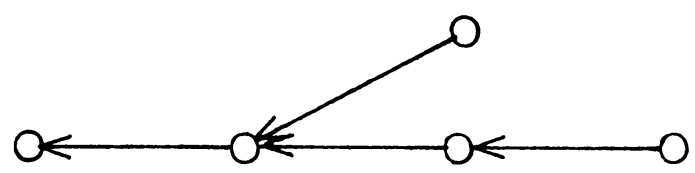

corresponds an $M$-set containing the configuration:

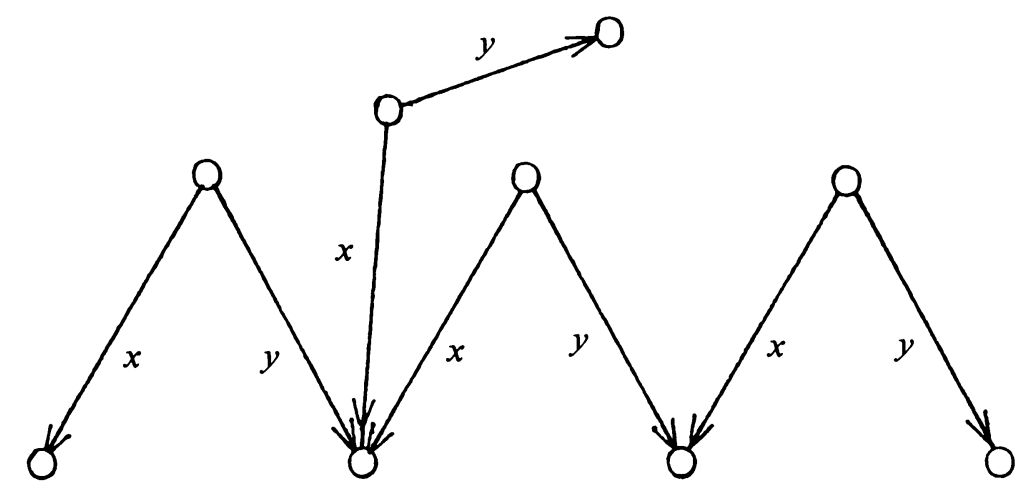

Such $M$-sets can be obtained from a disjoint union of copies of $M$ by way of an appropriate congruence relation; the conditions on $x$ and $y$ guarantee that no undesirable identifications will be made by this congruence. One sees inductively that these $M$-sets are irreducible, so $S(M)$ has no terminal object. 

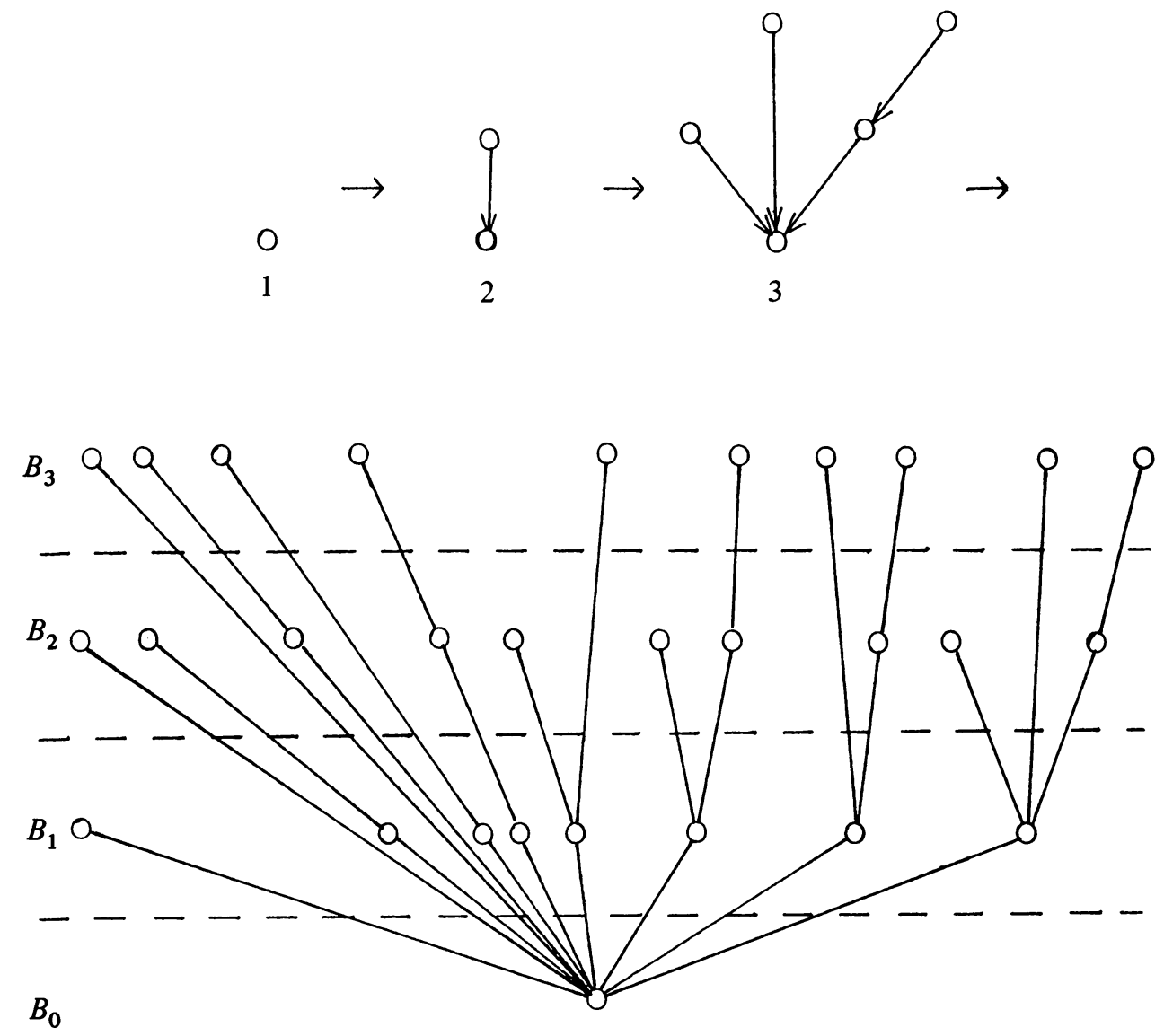

etc.

\section{FIGURE 3}

$3 \Rightarrow 1$ : We describe the construction of a cosolution set for each set $A$. Suppose $B$ is an $M$-set, with a set map $B \rightarrow A$. Without loss of generality, we may suppose $B$ is connected. Since $M$ is left ordered, its $\mathcal{L}$-classes [2, p. 47] are also ordered by the size of the left ideals they generate. Say these classes are $L_{0}, L_{1}, \ldots, L_{n}$, where $L_{i} M \subset L_{j} M$ for $i<j$. Since $M$ is left ordered, it also follows that $M$ is transitive on $B_{0}=L_{0} B$ (so $\left|B_{0}\right| \leq|M|$ ) and on each component of $B_{1}=L_{1} B-B_{0}$. Considering first those components into which $M$ sends no other points of $B$, we identify any two which map into $A$ the same way. Next one moves back to $B_{2}=L_{2} B-L_{1} B$, and again considers only components of $B_{2}$ isolated from higher levels. Those which $L_{1}$ sends into $B_{0}$ are treated as above. But if $L_{1}$ sends any component of $B_{2}$ into a component of $B_{1}$, we can only identify if both components (say $K$ and $L_{1} K$ ) map into $A$ the same way as do the other two (say $K^{\prime}$ and $L_{1} K^{\prime}$ ). In this way one works successively up one level higher and then back down, until $B_{n}$ is treated and the process stops. The cardinality of the resulting structure depends only on $M$ and $A$. 
REMARKS. 1. The last part of the proof also applies to infinite monoids that have only a finite number of $\mathcal{L}$-classes, but then $G(A)$ need no longer be finite for finite $A$.

2. The pattern of the "balayage" process described in the last part of the proof is best followed by working out an example or two. Consider for instance the construction of the maximal component of $T$ in the case where $M$ is a total order under the multiplication $x y=x \wedge y$ (see Figure 3).

3. The structures that appear as $G(A)$ are in general neither small nor simple; for example, when $M$ is a five-element total order, $T$ has about $4 \times 10^{627}$ elements.

\section{REFERENCES}

1. M. Barr and C. Wells, Toposes, triples, and theories, Grundlehren Math. Wiss., Bd. 278, Springer-Verlag, 1985.

2. A. H. Clifford and G. B. Preston, Algebraic theory of semigroups, Vol. 1, Math. Surveys, No. 7, Amer. Math. Soc., Providence, R. I., 1961.

3. Robert C. Davis, Combinatorial examples in universal coalgebra, Proc. Amer. Math. Soc. 89 (1983), 32-34.

4. (1984), 332-334.

Department of Mathematics, SOUThern Methodist University, Dallas, TEXAS 75275 